\title{
Polymer-directed Crystallization of Sibutramine using Cellulose Derivatives
}

\author{
Harim Bae, Hyeseung Lee, Minkyung Lee and Jonghwi Lee ${ }^{\dagger}$ \\ Department of Chemical Engineering and Materials Science, Chung-Ang University, 221, \\ Heukseok-dong, Dongjak-gu, Seoul 156-756, Korea
}

(Received December 2, $2010 \cdot$ Revised January 26, $2011 \cdot$ Accepted January 27, 2011)

\begin{abstract}
Nonclassical pathway of crystallization has been utilized to modify the properties and morphologies of inorganic and organic/inorganic materials. In here, the polymer-directed crystallization method has been applied to the pharmaceutical active ingredient to assess the applicability for as a particle engineering tool. The polymer-directed crystallization was successful to modifying the crystal size, habit and morphology, but it was not effective to discover the novel polymorphs of Sibutramine (SB). SB was selected as a model drug and polyacrylic acid (PAA), polyethylene imine (PEI) and chitosan (CHI) were added as a crystallization pathway modifier. SB was crystallized via drowning crystallization using methanol or ethanol as a solvent and water as a non-solvent. The significant interactions between polymer and the drug were confirmed by measuring the solubility of the drug in presence of polymer during the crystallization. The crystal forms of SB are characterized by X-ray diffraction (XRD), scanning electron microscope (SEM) and optical microscope (OM). The polymer-directed crystallization seems to be able to modify the crystal properties of pharmaceutical active ingredient, which is critical in determining the bioavailability, processability, and stability.
\end{abstract}

Key words - Sibutramine, Crystallization, Polymer-directed crystallization, Polymer drug interaction, Crystal habit

Crystal polymorphism is a key point in the research and development of drugs because it influence the final performance of dosage forms. For a long time, traditional methods of controlling the phenomenon of polymorphism(Price et al., 2002; Price et al., 2003; Rodriguez-Spong et al., 2004), for example, modification of crystallization, condition changing solvent, temperature of crystallization, extent of super saturation, or melting crystallization were used, but these ways were not very efficient to find new polymorphs.

Meanwhile, in the area of biomineralization, the polymerdirected crystallization has been successful to discover the novel polymorphs and has also facilitated the growth of single crystals of small molecules (Lang et al., 2002; Price et al., 2005), extended networks (Grzesiak et al., 2007), and inorganic complexes (Grzesial et al., 2007). This is very suitable for structural identification of both stable and 'metastable' polymorphs, because it casts light on the intermolecular interactions stabilizing the structure of crystals and gives unambiguous proof of the existence of a novel crystal form. A selfconsistent set of spectroscopic, calorimetric, and powder X-ray diffraction (PXRD) data can then be correlated with the corresponding structure allowing more accurate phase identifi-

Corresponding Author:

Tel : +82-2-816-5269, E-mail : jong@cau.ac.kr

DOI : 10.4333/KPS.2011.41.1.045 cation during manufacture (Adam et al., 2007).

Herein, the polymer-directed crystallization was applied to a model drug to assess the usefulness of the technique for modifying the properties of active ingredients. Sibutramine was used as a model drug, and its polymorphs were screened. Several polymers which have negative charge were chose for polymer-directed crystallization. The H-bonds originally formed between SB carbonyl and amine groups were broken Sibutramine carbonyl and amine groups and were replaced by those formed between Sibutramine and polymers in the drug-polymer composites. Using various material characterization techniques were applied to identify the influence of polymer in directing the crystallization pathway. These efforts will eventually combined with the current pharmaceutical researches such as solid dispersion, sustained release systems, gastric retention systems, etc.

\section{Materials and Methods}

\section{Materials}

Sibutramine (SB) is provided by AmorePacific Co (Seoul, Korea) and used without purification. Polyacrylic acid (PAA, $\mathrm{Mw}=14000 \mathrm{~g} / \mathrm{mol}, \mathrm{Mw}=45000 \mathrm{~g} / \mathrm{mol}$ ), polyethylene glycol (PEG, $\mathrm{Mw}=2000 \mathrm{~g} / \mathrm{mol}$ ) were purchased from Sigma-Aldrich (St. Louis., MO, USA). Chitosan (CHI, Mw $=3000 \mathrm{~g} / \mathrm{mol}$, $\mathrm{Mw}=10000 \mathrm{~g} / \mathrm{mol}$ ) was purchased from KITTOLIFE (Pyong- 
tack, Gyeonggi, South Korea). HPLC grade water (J.T. Baker, NJ, USA) was used without purification. As a solvent of drowning-out crystallization, methanol and ethanol (99.9\%, HPLC) were purchased from Samchun Pure Chemical (Pyongtack, Gyeonggi, South Korea).

\section{Crystallization of SB}

SB was first dissolved into methanol (30 wt $\%)$ and polymers were dissolved in water. The wt ratio of polymer : SB was fixed to be 1:9. A 96-well plate (polypropylene, $0.3 \mathrm{~mL}$, Bioneer Co., Korea) was used for drowning-out crystallization using methanol and ethanol as solvents, and acetone and water as non-solvents. The volume ratio of SB solution:non-solvent was $1: 9,2: 8,3: 7,5: 5,7: 3,8: 2,9: 1$, and the final volume of solvent and non-solvent mixture was $0.1 \mathrm{~mL}$. A thermal gradient system (MyGenie 96 Gradient Thermal Block, Bionner Co., Korea) was used to screen the initial crystallization conditions by changing the temperature $\left(25,35,45^{\circ} \mathrm{C}\right)$. After mixing with non-solvent for $1 \mathrm{~min}$ (Bortex, Scientific Industries, Inc., USA), crystallization was allowed at a constant temperature $\left( \pm 0.3^{\circ} \mathrm{C}\right)$ for $24 \mathrm{hrs}$. Unless otherwise stated, methanol and water were used as the solvent and the non-solvent, respectively, and the temperature and the volume ratio of SB solution:non-solvent were $25^{\circ} \mathrm{C}$ and $7: 3$.

\section{Characterization of particles}

After filtering and washing with water, crystallized particles were vacuum dried for more than $24 \mathrm{hrs}$ at R.T. The crystallinity and crystal structure of dried powders were detected by a X-ray powder diffractometer (XDS 2000, Scintag, USA) at a scan rate of 1 degree/min $\left(2 \theta: 10^{\circ}-40^{\circ}\right)$. To investigate morphology of particles, an optical microscope (OM, BX-51, Olympus, Japan) and a scanning electron microscope (SEM, S-3400, Hitachi, Japan) was used. The particles were mounted on the surface of carbon tape, and Pt coated by a carbon coater (Hitachi, Japan) at $6.7 \mathrm{~nm} / \mathrm{min}$ for $120 \mathrm{sec}$.

\section{Solubility analysis of SB}

The dissolved amount of SB was traced using a UV/visible spectroscopy (V-550, JASCO, Japan). SB $135 \mathrm{mg}$ was dissolved in methanol of $4.5 \mathrm{~mL}$ and water of $2.1 \mathrm{~mL}$ was input as a non-solvent. The rate of input was $0.6 \mathrm{ml} / \mathrm{h}$, and the final volume ratio of solvent and non-solvent was 7/3. After the non-solvent input, every $10 \mathrm{~min}$, the sample of $0.2 \mathrm{~mL}$ was extracted and its transparent supernatant $(0.1 \mathrm{~mL})$ was diluted by 1050 times with methanol, followed by UV/visible characterization. The absorption peak at $223 \mathrm{~nm}$ was quantified for the concentration results of $\mathrm{SB}$.

\section{Results and discussion}

\section{Crystal habits}

Crystal morphology plays a valuable role in pharmaceutical processing and product development of solid dosage forms. Differences in crystal habit may strongly influence the particle orientation, modify flowability, packing, compaction, compressibility and dissolution characteristics of a drug powder (Fig. 1). Solid-liquid interface interactions can alter the roundness of the interfaces, change crystal growth kinetics and enhance or inhibit growth at certain crystal faces, resulting in different habits (acicular, plates, tabular, bladed, prismatic, etc.) (Tiwary, 2001). Fig. 1 shows the nonclassical pathway of crystallization in presence of polymers dissolved in drug solutions. Polymers modify the surface energy of nuclei and growing crystals, and also the free energy of dissolved drug molecules, resulting in stabilizing mesocrystals.

Fig. 2 shows the effect of solvent on the crystallization of SB and the ratio of solvent vs. non-solvent. All the cases of OM images show that their shapes are irregular but mainly plate shaped crystals were banked up. Their average particle size can become larger in presence of polymers compared to the case of recrystallized SB. Thus, it might be the case that the polymers adsorbed on the surfaces of the SB crystals, which may or may not be fully grown, trigger the self assembly process of mesocrystals. The pale colors in the original micrographs taken under crossed polarizers proved the crystalline characters of crystallized SB.

Fig. 3 shows the particle morphology of polymer-directed

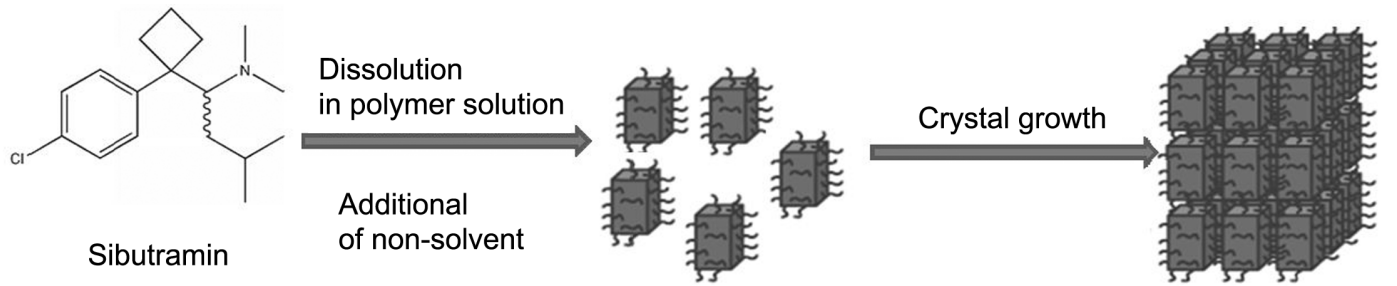

Figure 1. Schematic representation of mesocrystal formation via the polymer-directed drowning-out crystallization of Sibutramine (SB). 


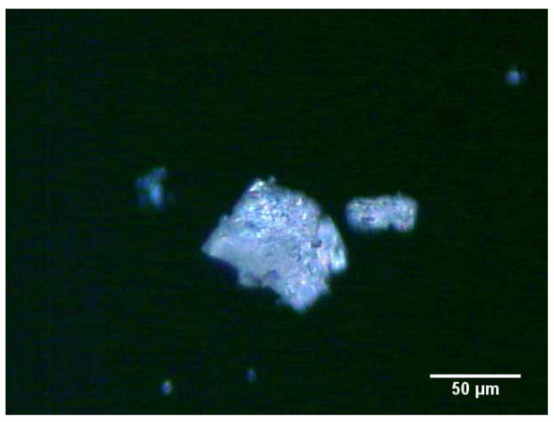

(a) Sibutramin (as received)

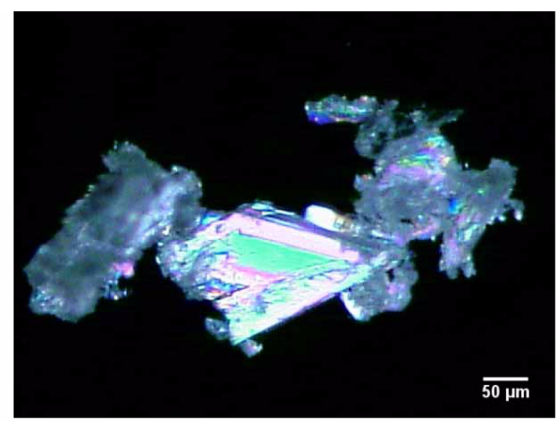

(b) $\mathrm{EtOH}(6: 4)$

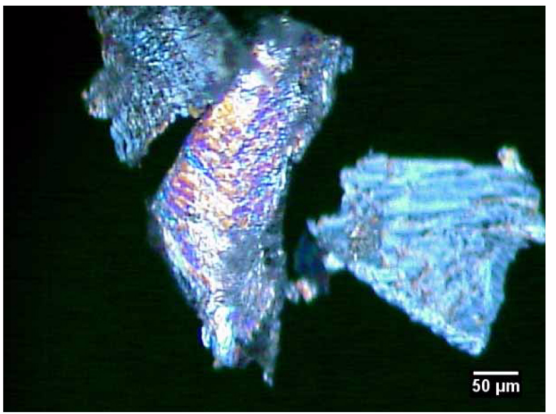

(c) $\operatorname{EtOH}(4: 6)$

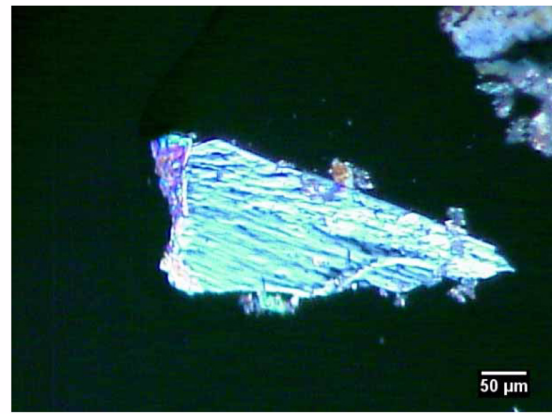

(d) $\mathrm{MeOH}(6: 4)$

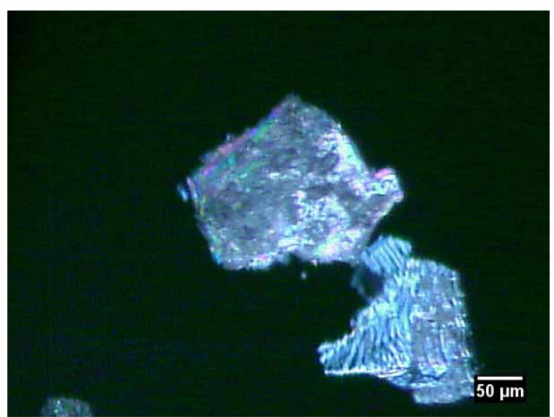

(e) $\mathrm{MeOH}(4: 6)$

Figure 2. Optical microscopy images (under crossed polarizers) of SB crystals obtained by drowning-out crystallization at $25^{\circ} \mathrm{C}$ using different solvents (solvent : nonsolvent volume ratio).

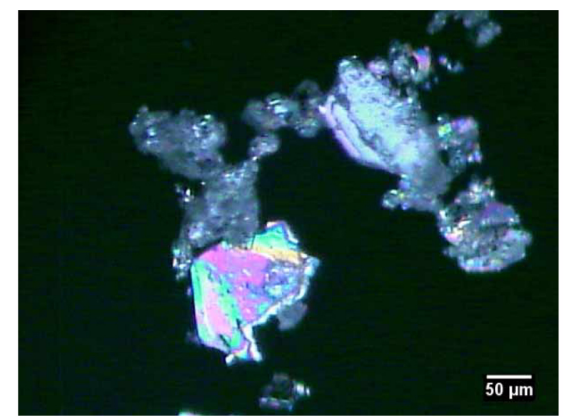

(a) Recrystallized sibutramine

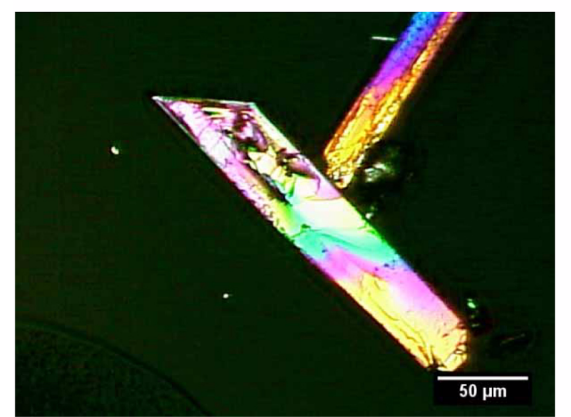

(d) PAA (Mw=45000)

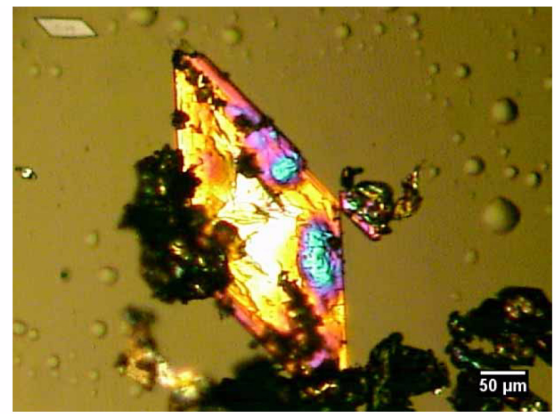

(b) PEI

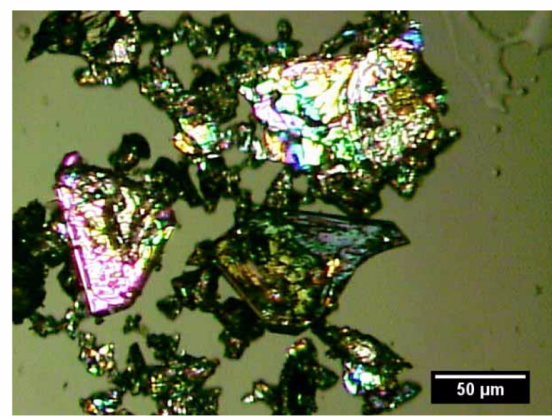

(e) $\mathrm{CHI}(\mathrm{Mw}=3000)$

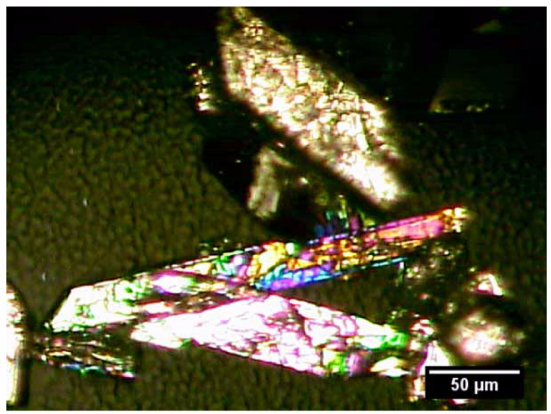

(C) PAA $(\mathrm{Mw}=1800)$

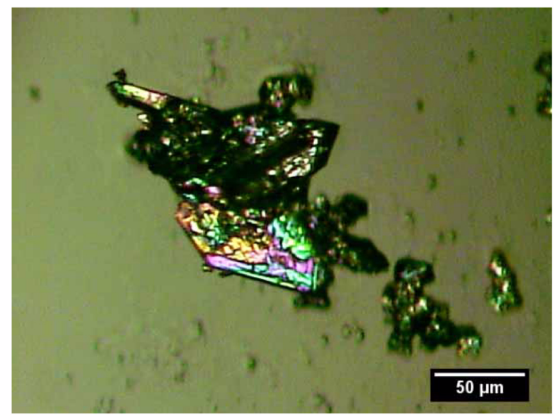

(f) $\mathrm{CHI}(\mathrm{Mw}=10000)$

Figure 3. Optical microscopy images (under crossed polarizers) of SB crystals obtained by drowning-out crystallization using different polymers.

crystallized SB in presence of various dissolved polymers by OM, and Fig. 4 shows the same samples by SEM. The role of the polymer on nucleation, growth, and assembly to a mesoc- rystal could be presumed. The pure SB shows white crystalline powder (Fig. 2a). The other particles show different morphologies depending on the type of polymers. The crystals 


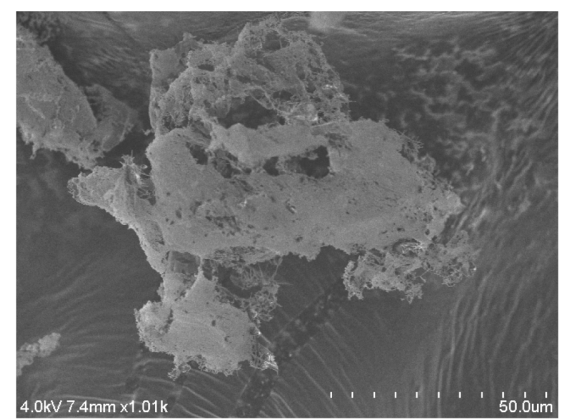

(a) Sibutramin(as received)

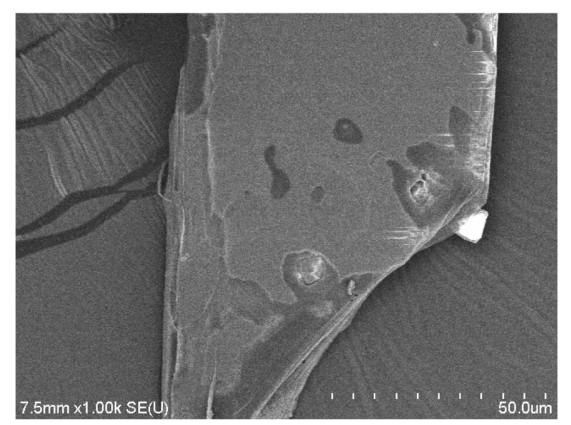

(d) PAA $(\mathrm{Mw}=45000)$

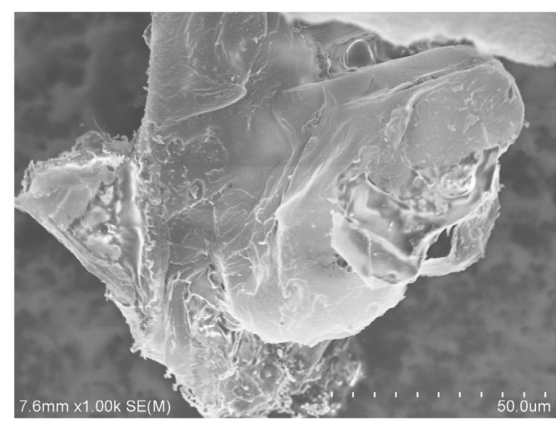

(b) PEI

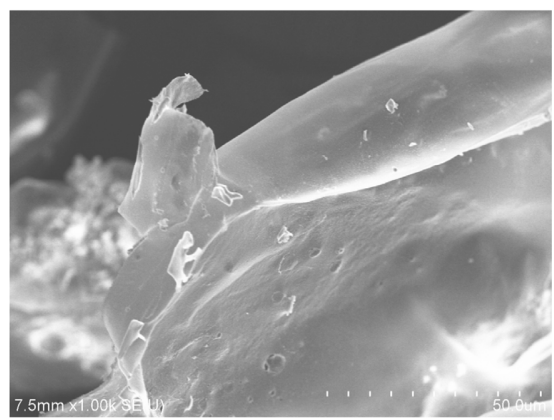

(e) $\mathrm{CHI}(\mathrm{Mw}=3000)$

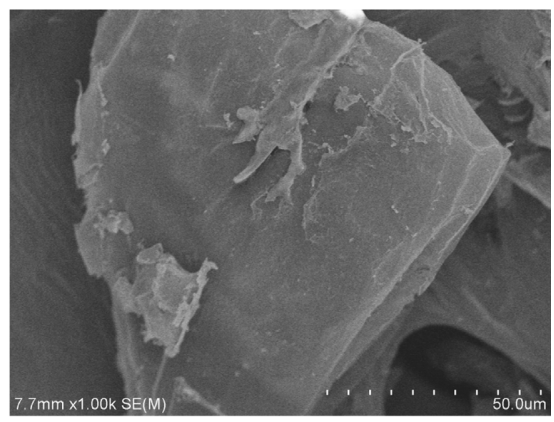

(c) PAA $(\mathrm{Mw}=1800)$

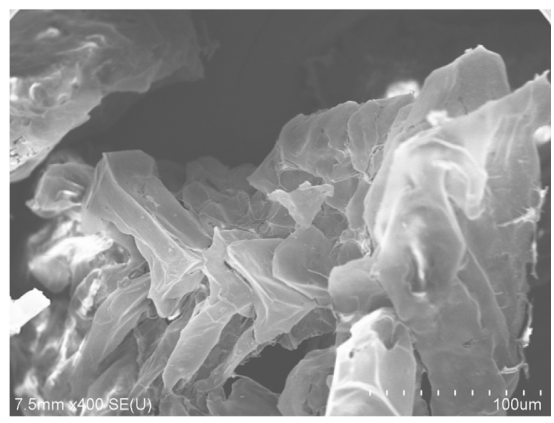

(f) $\mathrm{CHI}(\mathrm{Mw}=10000)$

Figure 4. SEM micrographs of polymer-directed SB crystals using different polymers.

with low and high molecular weight of PAA show plate morphology of large aspect ratios (Fig. 3c, 3d, 4c, and 4d) and those with low and high molecular weight of $\mathrm{CHI}$ show rather irregular but still assembled structures of plates of crystal (Fig. $3 e, 3 f, 4 e$, and $4 \mathrm{f}$ ). Polymers led to the change of crystal size and shape mainly as a result of the influence of the interfacial tension between nuclei and solution.

In the SEM observation, the crystal habit could not be clearly defined since the surfaces of particles are not quite regular. The main reason why the surfaces are not regular is that the surfaces are covered by the polymers. Therefore, the results prove that the nonclassical pathway is working to a certain extent.

\section{Crystal structures}

XRD is a sensitive foolproof method for solid-state characterization as the results are obtained directly from the molecular arrangements of the crystalline material (Chao and Vail, 1987). Fig. 5 and 6 shows the powder XRD results of SB, one from the cases of diverse solvents and the other from the cases of diverse polymers. We expected different polymorphs since the SEM images of SB with polymers were different. However, significant change in XRD pattern was not observed in the case with PAA, PEI and CHI. In all the cases, the major patterns were similar having only different relative peak inten- sity, which is mainly related with the orientation of crystalline domains. The existence of polymers influencing the crystallization pathway of SB did not modify the crystalline polymorph of SB, although it seems to influence the assembly structures of crystalline primary particles in mesocrystals.

\section{Solubility analysis of SB}

Since the XRD results did not disclose any influence of polymers, the existence of mesocrystals was only indirectly proved by the modification of crystal habits. For the activation of nonclassical pathway of crystallization proposed in Fig. 1 to play a role, the strong interactions between polymers and crystals are necessary. Therefore, another experimental proof of nonclassical pathway of crystallization was tried. As developed in the studies of self emulsifying drug delivery systems and solid dispersion, which focus on the prevention of recrystallization after dosing, if there is an enough interaction between a polymer and drug, the supersaturation criteria will be dependent on the existence of the polymer. Thus, the solubility change of SB in presence of a polymer could show an evidence of the interactions.

The solubility of SB with and without PAA $(\mathrm{Mw}=14000)$ was traced as a function of time lapse during crystallization. The solubility of SB in presence of PAA was always higher than that of SB (Fig. 7). The crystallization also starts at a rel- 


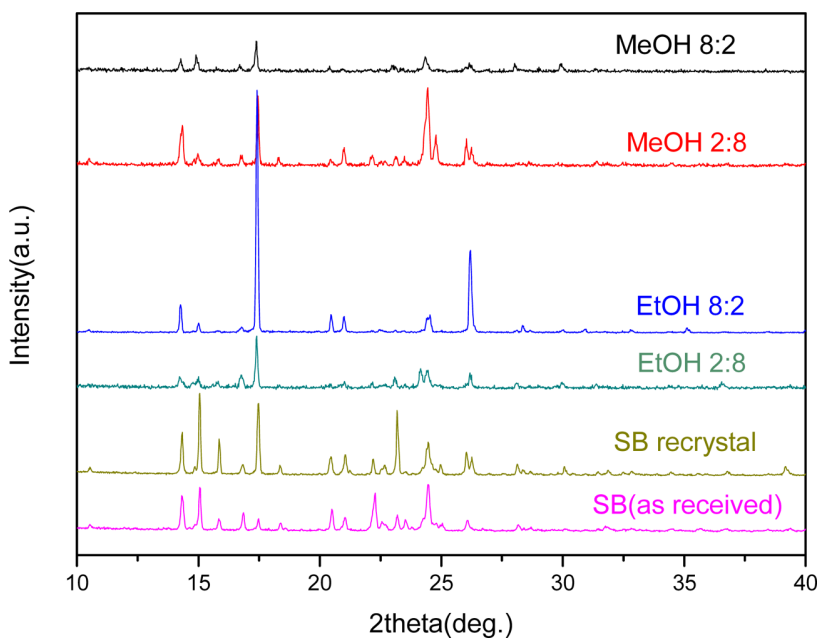

Figure 5. X-ray diffraction results of the effect of the solvent on the crystalline polymorphs of SB using different solvents (solvent : nonsolvent volume ratio).

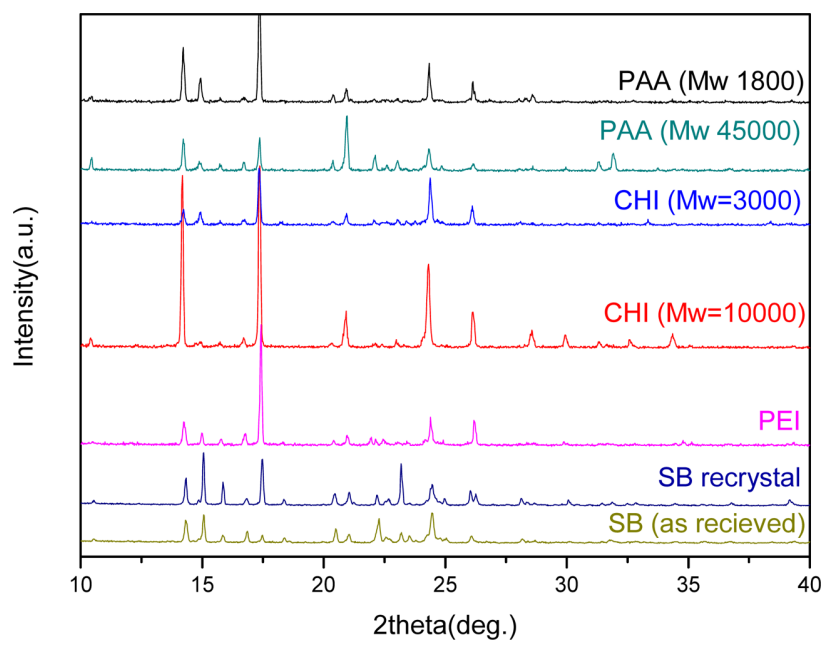

Figure 6. X-ray diffraction results of polymer-directed crystallized SB using different polymers.

atively higher SB concentration when PAA was dissolved together. Furthermore, the plateau region of the solubility curves shows the higher equilibrium solubility too. Solvate forms of crystals might result too. Inclusion of organic solvents in the solvated form tends to increase solubility and dissolution rate, presumably by weakening the crystalline lattice (Brittain and Grant, 1999)

\section{Conclusions}

As a nonclassical pathway of crystallization, polymerdirected crystallization was tried to explore its possibility as a particle engineering tool for pharmaceutical active ingredients. The results of SEM, PXRD, and polarized light optical micros-

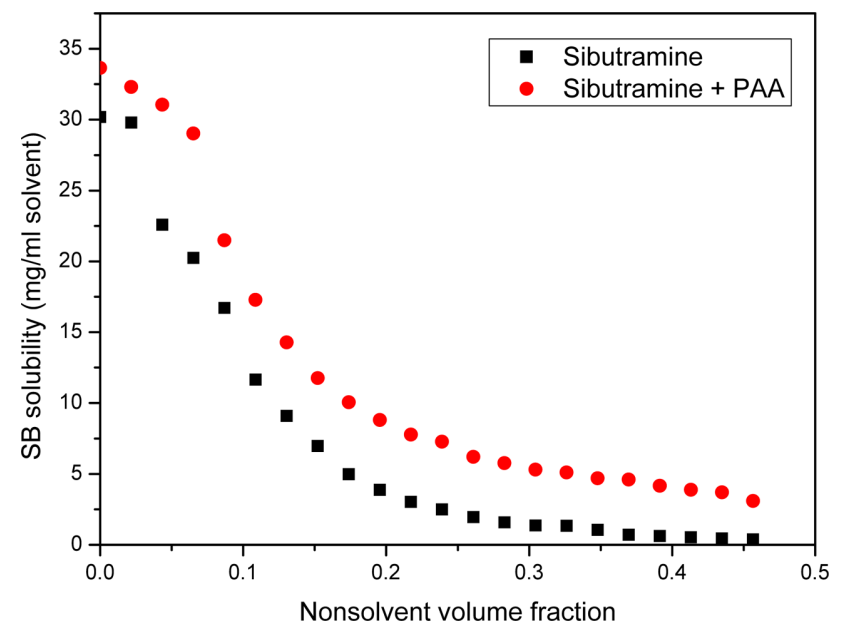

Figure 7. Effect of polymer addition on SB solubility as a function of non-solvent volume fraction.

copy demonstrated that a new crystal form of SB was not generated from the polymer-directed crystallization with PAA, PEI and CHI. Although different types of polymers resulted in different crystal habits, the concentration of polymer does not seem to be important for the formation of solid forms. However, when CHI was used for the polymer-directed crystallization, there was a significant difference in the morphology of crystals. Both the formation and the morphologies of crystals apparently contradict the classical crystallization, where growth of shapes is based on the integration of atomic or molecular building blocks into energetically favorable sites in a growing crystal face. The polymer-directed crystallization of SB in presence of PAA showed higher supercritical saturation concentration and delayed nucleation compared to the pure SB recrystallization. These results showed that the polymerdirected crystallization produced unique mesocrystals, and this method could be a simple and effective route to modify the properties of crystalline drugs, which makes the method useful in the pharmaceutical research and development.

\section{Acknowledgements}

This work was supported by the Seoul Development Institute (No. PA090732) and the Industrial Strategic Technology Development Program (No. 10035574) funded by the Ministry of Knowledge Economy (MKE, Korea). ML would like to thank the Human Resource Training Project for Strategic Technology (MKE and KOTEF).

\section{References}

Adam, L.G., Adam, J. M., 2007. New form discovery for the anal- 
gesics flurbiprofen and sulindac facilitated by polymerinduced heteronucleation. J. Pharm. Sci. 96, 2978-2986.

Brittain, H.G., Grant, D.J.W., 1999. Effects of polymorphism and solid-state solvation on solubility and dissolution rate. In: Swarbrick, J. (Ed.), Polymorphism in Pharmaceutical Solids. Marcel Dekker, New York, pp. 279-330.

Chao, R.S., Vail, K.C., 1987. Polymorphism of 1,2-dihydro-6-neopentyl-2-oxonicotinic acid: characterization, interconversion, and quantitation. Pharm. Res. 4, 429-432.

Grzesiak, A.L., Matzger, A.J., 2007. Selection and discovery of polymorphs of platinum complexes facilitated by polymerinduced heteronucleation. Inorg. Chem. 46, 453-457.

Grzesiak, A.L., Uribe, F.J., Ockwig, N.W., Yaghi, O.M.,Matzger, A.J., 2006. Polymer-induced heteronucleation for the discovery of new extended solids. Angew. Chem. Int. Edit 45, 25532556.

Lang, M., Grzesiak, A.L., Matzger, A.J., 2002. The use of polymer heteronuclei for crystalline polymorph selection. J. Am. Chem. Soc. 124, 14834-14835.

Price, C.P., Grzesiak, A.L., Kampf, J.W., Matzger, A.J., 2003. Maize 1: A trimorphic azo pigment. Cryst. Growth Des. 3, 1021-1025.

Price, C.P., Grzesiak, A.L., Lang, M., Matzger, A.J., 2002. Polymorphism of abumetone. Cryst. Growth Des. 2, 501-503.

Price, C.P., Grzesiak, A.L., Matzger, A.J., 2005. Crystalline polymorph selection and discovery with polymer heteronuclei. J. Am. Chem. Soc. 127, 5512-5517.

Rodríguez-Spong, B., Price, C.P., Jayasankar, A., Matzger, A.J., Rodríguez-Hornedo, N., 2004. Generalprinciples of pharmaceutical solid polymorphism: A supramolecular perspective. Adv. Drug Delivery Rev. 56, 241-274.

Tiwary, A.K., 2001. Modification of crystal habit and its role in dosage form performance. Drug Dev. Ind. Pharm. 27, 699-709. 